\title{
Effet du mélange de pelure de banane plantain et de compost de fiente de poules sur la croissance en pépinière de rejets écailles de bananier plantain, variété Big Ebanga (Musa AAB sg Plantain)
}

BOMISSO Edson Lezin ${ }^{1}$, OUATTARA Généfol'1, TUO Seydou ${ }^{1 *}$, ZELI Tizié Fabrice ${ }^{1}$ et AKÉ Séverin ${ }^{1}$ 1 Université Félix HOUPHOUËT-BOIGNY, UFR Biosciences, Laboratoire de Physiologie Végétale, 22 BP 582 Abidjan 22, Côte d'Ivoire.

Auteur correspondant : E-mail : tuoseydou4@yahoo.fr ; tél : +22508699756

Original submitted in on $21^{\text {st }}$ September 2018. Published online at www.m.elewa.org on $31^{\text {st }}$ October 2018 https://dx.doi.org/10.4314/jab.v130i1.1

\section{RÉSUMÉ}

Objectif : La présente étude visait à trouver une alternative à l'utilisation des engrais de synthèse dans la culture du bananier plantain.

Méthodologie et résultats : Pour ce faire, des formulations d'engrais organiques ont été mises au point. Notamment, F2 constitués de $100 \%$ de pelure de banane séchée et broyée (PBSB), F3, F4 et F5 constituées respectivement de $75 \%, 50 \%, 25 \%$ de PBSB $+25 \%, 50 \%$ et $75 \%$ de compost de fiente de poules (CFP). Les propriétés chimiques de ces formulations ont été déterminées, puis leur effet sur la croissance des rejets écailles de bananier plantain a été évalué comparativement à l'effet du NPK (20-10-10) et le témoin F0. L'expérimentation a été réalisée selon un dispositif en bloc de Fisher comportant 6 traitements avec 4 répétitions. Les applications ont été faites chaque mois et le suivi a porté sur la croissance en hauteur, la circonférence du pseudo-tronc et le nombre de feuilles. L'analyse minérale a permis de classer les fertilisants organiques en deux groupes : les plus riches (F4 et F5) et les moyennement riches (F2 et F3). Concernant les données de croissance, l'analyse statistique indique que les fertilisants organiques ont induit une bonne croissance $(\mathrm{H}=13,77 \mathrm{~cm}, \mathrm{C}=2,93 \mathrm{~cm}, \mathrm{NF}=8)$ des plants de bananiers par rapport au témoin $\mathrm{F0}(\mathrm{H}=8,45$ $\mathrm{cm}, \mathrm{C}=1,55 \mathrm{~cm}, \mathrm{NF}=7$ ) et à l'engrais minéral $(\mathrm{H}=6 \mathrm{~cm}, \mathrm{C}=-0,2 \mathrm{~cm}, \mathrm{NF}=5)$.

Conclusion et application des résultats : Cette étude a montré que l'utilisation de la pelure de banane plantain séchée et broyée (PBSB) simple ou en mélange avec le compost de fiente de poules (CFP) induit une croissance harmonieuse du bananier plantain. La PBSB peut être une alternative à l'utilisation des engrais chimiques et être recommandée aux agriculteurs.

Mots clés : Banane plantain, pelure, engrais organiques, engrais minéral 
Bomisso et al., J. Appl. Biosci. 2018 Effet du mélange de pelure de banane plantain et de compost de fiente de poules sur la croissance en pépinière de rejets écailles de bananier plantain, variété Big Ebanga

Effect of plant banana peel and chicken dung compost on growth in nursery of banana plantain plantlets, variety Big Ebanga (Musa AAB sg Plantain)

\begin{abstract}
Objective: The present survey aimed to find an alternative to the use of the mineral manures in the culture of the banana tree plantain.

Methodology and results: For that to make, formulations of organic manures have been finalized. Notably constituted F2 of $100 \%$ of banana peel dried and ground (PBSB), F3, F4 and constituted F5 respectively of $75 \%, 50 \%, 25 \%$ of PBSB $+25 \%, 50 \%$ and $75 \%$ of compost of droppings of hens (CFP). The chemical properties of these formulations have been determined, and then their effect on the growth of the dismissals scales of banana trees plantain has been valued compared to the effect of the NPK (20-10-10) and witness F0. The experimentation has been achieved according to a device in block of Fisher including 6 treatments with 4 repetitions. The applications have been made every month and the follow-up was about the growth in height, the circumference of the pseudo-trunk and the number of leaves. The mineral analysis permitted to classify the fertilizing organic in two groups: the richest (F4 and F5) and the rich (F2 and F3). Concerning the data of growth, the statistical analysis indicates that the fertilizing organic led a good growth $(\mathrm{H}=13.77 \mathrm{~cm}, \mathrm{C}=2.93$ $\mathrm{cm}, \mathrm{NF}=8)$ of the plantations of banana trees in relation to witness $\mathrm{FO}(\mathrm{H}=8.45 \mathrm{~cm}, \mathrm{C}=1.55 \mathrm{~cm}, \mathrm{NF}=7)$ and to the mineral manure $(\mathrm{H}=6 \mathrm{~cm}, \mathrm{C}=-0.2 \mathrm{~cm}, \mathrm{NF}=5)$.

Conclusion and application of results: This survey permitted to show that the use of the peel dried banana plantain and ground (PBSB) simple or in mixture with the compost of droppings of hens (CFP) induced a harmonious growth of the banana tree plantain. The PBSB can be an alternative to the use of the chemical manures and can recommend to the agriculturists.
\end{abstract}

Keywords: Banana plantain, peel, organic manures, mineral manures.

\section{INTRODUCTION}

Les bananiers (Musa spp.) sont originaires d'Asie du Sud-Est et sont cultivés dans plus de 120 pays du monde (Jones, 2000). Ils jouent un rôle important dans la sécurité alimentaire mondiale. Leurs fruits occupent le quatrième rang mondial des denrées alimentaires les plus importantes après le riz, le blé et le lait (FAO, 2010). La production mondiale de la banane plantain en 2016 était estimée à 35063802 millions de tonnes (FAOstat, 2017). Le bananier plantain appartient au sous-groupe plantain du groupe $A A B$ du genre Musa de la famille des Musacées et compte plus de 200 espèces cultivées dans le monde (Lassois et al., 2009 ; CNRA, 2016). La banane plantain est la principale source alimentaire de nombreuses populations à travers le monde et particulièrement dans les pays tropicaux (Jones, 2000 ; Lassois et al., 2009). Dans la sousrégion ouest africaine, la banane plantain occupe une place prépondérante dans l'alimentation des populations. En Côte d'Ivoire, le bananier plantain occupe le troisième rang parmi les cultures vivrières en termes de tonnages après l'igname, le manioc avec une production annuelle en 2016 d'environ 1,6 millions de tonnes (FAOstat , 2017). II constitue une source de revenus importante, d'emplois et de recette d'exportation (Foure et Tezenas, 2000). II joue également un rôle important en phytothérapie (Rabbani et al., 2001). Malgré cette importante production, l'accroissement démographique très rapide, entraine une demande sans cesse croissante de la banane plantain [120 Kg /personne/an, (Yao et al., 2014)]. Cette demande est largement supérieure à l'offre, résultant d'une production insuffisante et saisonnière. La problématique de sa production est liée à de nombreuses contraintes dont l'appauvrissement des sols cultivables. Pour pallier à cette difficulté, des agriculteurs utilisent des fertilisants chimiques qui améliorent la productivité des cultures (Diallo, 2002). Ainsi, l'utilisation de ces engrais chimiques, de par leur action bénéfique immédiate sur la productivité des cultures vivrières est une des solutions alternatives. Toutefois, leur coût élevé et leur indisponibilité les rendent presque inaccessibles aux petits paysans (Useni et al., 2012). 
Outre les problèmes écologiques et environnementaux qu'elle cause, la fertilisation minérale seule ne permet pas de maintenir la fertilité des sols. Son utilisation exclusive entraine une augmentation de l'acidité, une dégradation du statut physique et une baisse de la matière organique du sol (Mulaji, 2011). De plus, leur production et leur transport contribuent à l'émission des gaz à effets de serre (FAO, 2010). Dans un tel contexte, la fertilisation organique pourrait constituer une solution appropriée pour la restauration de la fertilité des sols et l'amélioration de la productivité du bananier plantain. Les pratiques de l'agriculture organique visent à augmenter la biodiversité et l'activité biologique des sols. Elles permettent ainsi d'atteindre les systèmes naturels optimaux qui sont socialement, écologiquement et économiquement durables (Yerima et al., 2014). De nombreux travaux ont montré que les résidus végétaux et les fumiers organiques comme la fiente de poules, sous-produits d'élevage contribuent à la croissance des plantes à travers leurs effets bénéfiques sur les propriétés physiques, chimiques et biologiques des sols (Aowad et Mohamed, 2009). Par contre, l'utilisation des amendements organiques tels que le mélange des produits végétaux et animaux est peu documentée dans notre pays, alors qu'ils constituent

\section{MATERIEL ET METHODES}

Matériel végétal : Le matériel végétal utilisé est constitué de rejets écailles de bananiers plantain de la variété Big Ebanga. Ces rejets proviennent de la plantation Dougba sise dans la localité d'Azaguié. Ils sont considérés comme de bons semenceaux pour la mise en place des exploitations bananières (Turquin et al., 2007).

Matériel fertilisant: Dans le cadre de cette étude, le matériel fertilisant a été constitué d'engrais minéral NPK (20-10-10), de compost de fiente de poules (CFP) et de pelure de banane plantain séchée et broyée (PBSB). La PBSB a été produite à partir de pelures de banane plantain collectées auprès des vendeuses sur les différents marchés de la ville d'Abidjan puis séchées avant d'être transformées en poudre au moulin.

\section{Méthodes}

Composition des formulations d'engrais organiques : Les effets de six traitements ont été évalués au cours de une source optimale d'éléments nutritifs pour les plantes (Mulaji, 2011). Ces dernières ont besoin essentiellement et en quantités importantes d'azote $(\mathrm{N})$, de phosphore $(\mathrm{P})$ et de potassium $(\mathrm{K})$ pour accomplir leur cycle de croissance. Le réapprovisionnement périodique des réserves du sol en ces éléments afin de maintenir une bonne productivité des cultures est ainsi indispensable (Moughli, 2000). Vu sa disponibilité en milieu urbain, la pelure de banane plantain séchée et broyée, utilisée simple ou en mélange avec le compost de fiente de poules pourrait constituer une alternative à l'utilisation des engrais minéraux. Pour une amélioration de la productivité du bananier plantain de manière durable et écologique, la présente étude se propose d'évaluer les effets de mélanges de pelure de banane et de compost de fiente de poules sur la croissance en pépinière des rejets écailles de bananiers plantain. Les objectifs spécifiques sont : (i) la détermination des caractéristiques chimiques des formulations à base de pelure de banane plantain séchée et broyée (PBSB) et de compost de fiente de poules (CFP), (ii) l'évaluation de l'effet des formulations sur les paramètres de croissance des plants de bananiers plantain et (iii) l'identification de la formulation qui favorise le développement harmonieux des bananiers plantain.

cette étude (Tableau 1). Suivant le traitement, un plant reçoit $50 \mathrm{~g}$ du mélange fertilisant.

Détermination de la composition minérale et des propriétés chimiques des formulations organiques Dosage des minéraux contenus dans les fertilisants organiques: Le sodium $(\mathrm{Na})$, le potassium $(\mathrm{K})$ et le calcium ( $\mathrm{Ca}$ ) ont été dosés par spectrophotométrie de flamme après incinération et minéralisation à l'acide chlorhydrique. L'acide phosphorique a été dosé colorimétriquement sur la solution chlorhydrique des cendres grâce à la coloration jaune qu'il donne avec le réactif vanadomolybdique. L'azote total a été dosé par la méthode de Kjéldhal (Goyal et al., 2005). Le magnésium a été dosé par spectrométrie d'absorption atomique à une longueur d'onde de $285 \mathrm{~nm}$. Les quantités de ces différents éléments ont été exprimées en \%. 
Tableau 1: Traitements appliqués aux plantules de bananier plantain.

\begin{tabular}{l|l|}
\hline Traitements & Compositions des fertilisants \\
\hline F0 & Sans apport de fertilisant \\
\hline F1 & NPK $(20-10-10)$ \\
\hline F2 & PBSB \\
\hline F3 & $75 \%$ PBSB $(37,5 \mathrm{~g})+25 \%$ CFP $(12,5 \mathrm{~g})$ \\
\hline F4 & $50 \%$ PBSB $(25 \mathrm{~g})+50 \%$ CFP $(25 \mathrm{~g})$ \\
\hline F5 & $(12,5 \mathrm{~g})+75 \%$ CFP $(37,5 \mathrm{~g})$. \\
\hline
\end{tabular}

$\mathbf{F 0}=$ témoin (terre tout venant) ; F2 = pelure de banane séchée et broyée ; F3 = 75\% F2 + $25 \%$ compost de fiente de poules ; F4 = $50 \% \mathrm{~F} 2+50 \%$ compost de fiente de poules ; F5 = $25 \% \mathrm{~F} 2+75 \%$ compost de fiente de poules ; PBSB : Pelure de banane plantain séchée et broyée ; CFP : Compost de fiente de poules.

\section{Caractérisation chimique des formulations organiques \\ Matières organique et sèche, $\mathrm{pH}$ et rapport $\mathrm{C} / \mathrm{N}$ : Le} taux de matière sèche est complémentaire du degré d'humidité (M'sadak et al., 2015). II s'est agi ainsi de déterminer le degré d'humidité qui est le pourcentage d'eau dans la matière considérée. II a été estimé après séchage à l'étuve des échantillons de sol à $70^{\circ} \mathrm{C}$ pendant $48 \mathrm{~h}$. La matière organique a été évaluée par la méthode de perte au feu. Une dizaine de gramme de chaque échantillon a été calcinée à $600^{\circ} \mathrm{C}$ pendant $5 \mathrm{~h}$ au four à moufle (Igoud et al., 2002). Le pH des mélanges fertilisants a été mesuré à l'aide d'un pH-mètre à lecture directe. Le rapport $\mathrm{C} / \mathrm{N}$ de ces mélanges a été déterminé par calcul.

Identification du mélange induisant une croissance harmonieuse des rejets
Calibrage et plantation des rejets écailles : Les rejets ont été prélevés sur le pied-mère et pesés. Puis, un calibrage a permis de ne retenir que des plants de poids compris entre 120 et $350 \mathrm{~g}$. Dans le cadre de cette étude, le substrat de culture a été prélevé au Centre National de Floristique de I'Université Félix Houphouët-Boigny et stérilisé deux (2) fois à l'autoclave pendant $2 \mathrm{~h}$ à $120^{\circ} \mathrm{C}$ par intervalle de $24 \mathrm{~h}$. Les seaux de $5 \mathrm{~L}$ ont été remplis au $4 / 5$ avec ce sol stérile. Le substrat a été arrosé abondamment avec de l'eau de robinet $24 \mathrm{~h}$ avant la mise en terre des rejets écailles sélectionnés.

Dispositif expérimental : Le dispositif qui a soutenu ces essais a été en blocs de Fisher complètement randomisés avec 6 traitements en raison de 4 répétitions par traitement (Figure 1). Chaque traitement a comporté 4 rejets écailles de bananier plantain, soit un total de $6 \times 4 \mathrm{x}$ $4=96$ rejets. Les écarts ont été entre les traitements et entre les blocs, respectivement de 20 et $50 \mathrm{~cm}$. 


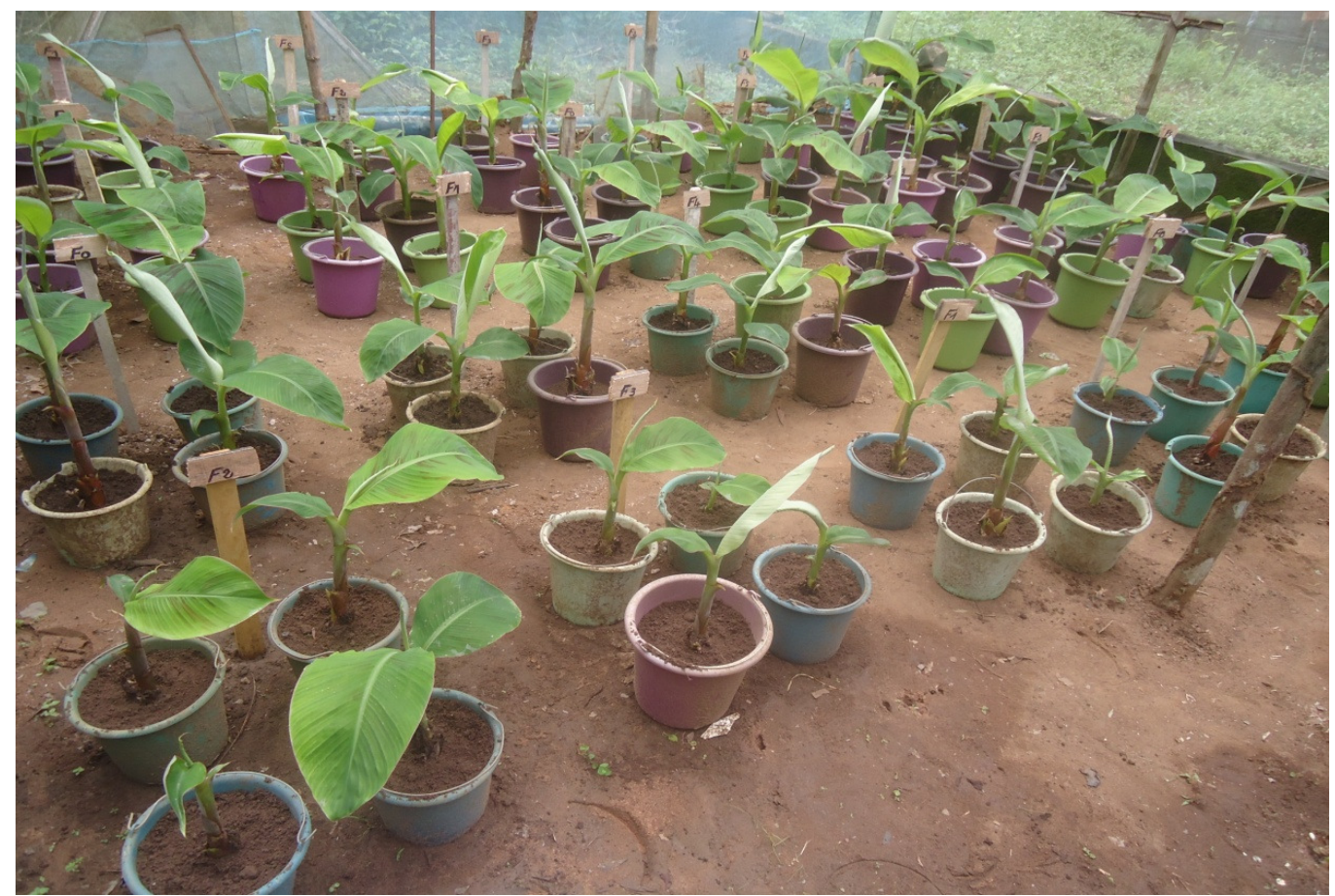

Figure 1 : Dispositif expérimental en bloc complètement randomisé

Fertilisation et entretien : Les rejets mis en terre ont été arrosés tous les deux jours avec de l'eau de robinet. Quatre semaines après la mise en terre, les premiers traitements ont été appliqués aux jeunes plantes. Une dose de $50 \mathrm{~g}$ de chaque formulation a été apportée. Un mois après, les mêmes doses de fertilisant ont été apportées aux jeunes plantes de bananier plantain. Cette étude s'est déroulée sous abri sur une période de 4 mois (septembre à décembre 2017) et répétée de janvier à avril 2018. Les observations ont porté sur quelques paramètres de croissance.

Paramètres évalués : Les mesures de croissance ont été exprimées en centimètre. Les organes ayant fait l'objet de suivi ont été les feuilles et le pseudo-tronc.

Hauteur du pseudo-tronc $(\mathrm{H})$ : la hauteur du pseudotronc a été mesurée à partir du collet jusqu'au sommet de la plante au niveau du $\mathrm{V}$ que forment les deux dernières feuilles fonctionnelles à l'aide d'un ruban mètre, toutes les deux semaines.

Circonférence du pseudo-tronc (C) : elle a été évaluée à $4 \mathrm{~cm}$ au-dessus du niveau du sol à l'aide d'un ruban mètre, toutes les deux semaines.

\section{RESULTATS}

Composition minérale et propriétés chimiques des formulations

Teneurs en minéraux de chaque fertilisant : L'analyse des résultats relatifs aux teneurs en sodium ( $\mathrm{Na}$ ), azote
Nombre de feuilles (NF) : la croissance foliaire a été déterminée toutes les deux semaines en comptant le nombre de feuilles émises du bas vers le haut.

Robustesse $(\mathbf{H} / \mathbf{C})$ : c'est un paramètre qui permet d'évaluer la vigueur des plantules. Elle a été déterminée par le rapport Hauteur/ Circonférence.

Analyses statistiques: Les données de teneur en minéraux, de $\mathrm{pH}$ et de matière sèche de chaque formulation ont subi une analyse de variance à un critère de classification (ANOVA 1). Les données de croissance ont été soumises à une analyse de variance à effets principaux. L'hypothèse d'égalité des moyennes a été évaluée au risque $a=5 \%$. La comparaison des moyennes a été réalisée à l'aide du test de NewmanKeuls. L'analyse en composantes principales (ACP) a été réalisée en vue de caractériser les différents fertilisants sur la base de leurs teneurs en minéraux et de leurs effets sur la croissance des jeunes plantes de bananiers plantains. L'ensemble des tests a été réalisé à l'aide des logiciels Statistica version 7.1 et XLSTAT.

$(\mathrm{N})$, phosphore $(\mathrm{P})$, potassium $(\mathrm{K})$, calcium $(\mathrm{Ca})$ et magnésium $(\mathrm{Mg})$ des différents mélanges (Tableau 2) montre une différence significative $(p<5 \%)$ entre les valeurs moyennes des fertilisants. Le fertilisant F4 
présente les teneurs les plus élevées en sodium, phosphore et azote dont les valeurs sont respectivement $0,03 \% ; 1,34 \%$ et $2,04 \%$. Le fertilisant $\mathrm{F} 5$ est plus riche en sodium $(0,03 \%)$, magnésium $(0,27 \%)$ et en calcium $(0,85 \%)$. Pour le potassium, c'est le fertilisant F2 qui renferme la plus grande teneur dont la valeur est $2,51 \%$. L'analyse en composantes principales a permis de caractériser les fertilisants sur la base de leurs teneurs en minéraux (Figure 2). Le cercle de corrélation montre que les axes 1 et 2 contribuent pour $92,95 \%$ à la variation totale observée et que l'ensemble des minéraux sont positivement corrélés à l'axe 1 . La dispersion des fertilisants dans le plan 1 et 2 a permis d'obtenir trois (3) groupes de fertilisants dont : les plus riches (F4 et F5), les moyennement riches (F2 et F3) et les pauvres (F0) en minéraux.

Tableau 2: Teneur (\%) en Na, N, Ca, $\mathrm{P}, \mathrm{K}$ et Mg des différents traitements.

\begin{tabular}{l|l|l|l|l|l|l}
\hline Traitements & Azote (N) & $\begin{array}{l}\text { Calcium } \\
\text { (Ca) }\end{array}$ & $\begin{array}{l}\text { Magnésium } \\
(\mathbf{M g})\end{array}$ & $\begin{array}{l}\text { Phosphore } \\
\text { (P) }\end{array}$ & $\begin{array}{l}\text { Potassium } \\
\text { (K) }\end{array}$ & Sodium (Na) \\
\hline F0 & $1,67 \mathrm{c}$ & $0,6 \mathrm{e}$ & $0,22 \mathrm{bc}$ & $0,25 \mathrm{~d}$ & $0,03 \mathrm{e}$ & $0,00 \mathrm{c}$ \\
\hline F2 & $1,96 \mathrm{~b}$ & $0,69 \mathrm{~d}$ & $0,2 \mathrm{c}$ & $0,3 \mathrm{~cd}$ & $2,51 \mathrm{a}$ & $0,022 \mathrm{~b}$ \\
\hline F3 & $1,92 \mathrm{~b}$ & $0,71 \mathrm{c}$ & $0,22 \mathrm{bc}$ & $0,5 \mathrm{c}$ & $2,26 \mathrm{~b}$ & $0,024 \mathrm{ab}$ \\
\hline F4 & $2,04 \mathrm{a}$ & $0,74 \mathrm{~b}$ & $0,25 \mathrm{ab}$ & $1,34 \mathrm{a}$ & $1,91 \mathrm{c}$ & $0,03 \mathrm{a}$ \\
\hline F5 & $1,94 \mathrm{~b}$ & $0,85 \mathrm{a}$ & $0,27 \mathrm{a}$ & $0,86 \mathrm{~b}$ & $1,37 \mathrm{~d}$ & $0,03 \mathrm{a}$ \\
\hline
\end{tabular}

Dans une même colonnes les moyennes suivies de la même lettre ne diffèrent pas significativement selon le test de NewmanKeuls au seuil de $5 \%$.

$\mathbf{F 0}=$ témoin (terre tout venant) ; F2 = pelure de banane séchée et broyée ; F3 = 75\% F2 + $25 \%$ compost de fiente de poules ; F4 = $50 \%$ F2 + $50 \%$ compost de fiente de poules ; F5 = $25 \%$ F2 + $75 \%$ compost de fiente de poules.

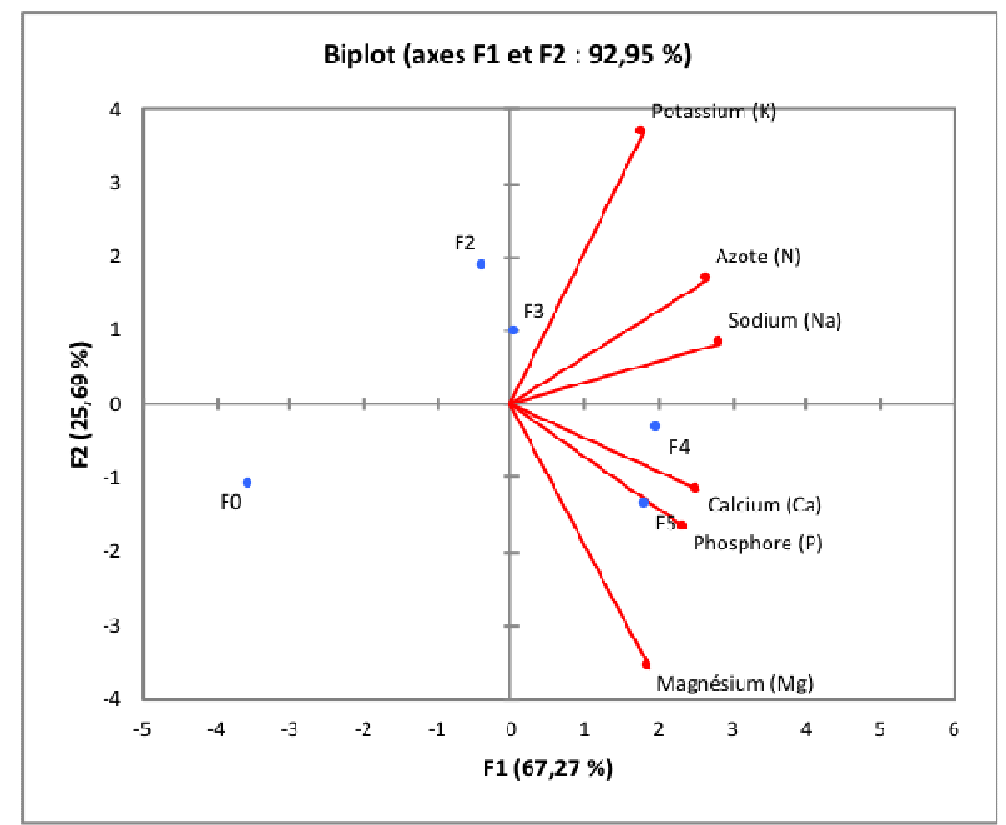

Figure 2 : Répartition des fertilisants et des paramètres de caractérisation de la composition minérale dans le plan 1et 2 d'une analyse en composante

\section{Propriétés chimiques des formulations}

pH: L'analyse des valeurs moyennes de potentiel d'hydrogène (Tableau 3) montre que celles-ci se situent entre 6,2 et 9,2. La différence notée entre ces moyennes a été significative $(p<5 \%)$. En tenant compte de l'échelle des $\mathrm{pH}$, deux groupes de fertilisants se distinguent : acide dont F0 $(6,2)$ et basique, dont le plus basique est le fertilisant $F 2(9,2)$.

Matières sèche et organique, carbone organique et rapport $\mathbf{C} / \mathbf{N}$ : Le tableau 3 présente les données relatives aux caractéristiques chimiques de chaque fertilisant. L'analyse de ce tableau montre une différence 
significative entre les moyennes $(p<5 \%)$. Le fertilisant F2 est beaucoup plus riche en matière organique $(75,85$

$\%)$ et en carbone organique $(58,11 \%)$. Ce dernier possède un rapport $\mathrm{C} / \mathrm{N}$, très élevé, de l'ordre de 27,2 . Tandis que le témoin F0 présente un meilleur taux de matière sèche $(93,5 \%)$ et un faible ratio carbone/ azote.

Tableau 3: Propriétés chimiques des différents traitements.

\begin{tabular}{l|l|l|l|l|l}
\hline Traitements & pH & MS (\%) & MO (\%) & Corg & C/N \\
\hline F0 & $6,17 \mathrm{e}$ & $93,5 \mathrm{a}$ & $4,84 \mathrm{e}$ & $2,39 \mathrm{e}$ & $1,4 \mathrm{e}$ \\
\hline F2 & $9,17 \mathrm{a}$ & $87,62 \mathrm{~d}$ & $75,85 \mathrm{a}$ & $58,11 \mathrm{a}$ & $27,2 \mathrm{a}$ \\
\hline F3 & $8,93 \mathrm{~b}$ & $89,39 \mathrm{c}$ & $65,1 \mathrm{~b}$ & $45,42 \mathrm{~b}$ & $23,7 \mathrm{~b}$ \\
\hline F4 & $8,73 \mathrm{c}$ & $89,79 \mathrm{c}$ & $53,83 \mathrm{c}$ & $37,38 \mathrm{c}$ & $18,3 \mathrm{c}$ \\
\hline F5 & $7,8 \mathrm{~d}$ & $92,24 \mathrm{~b}$ & $40,52 \mathrm{~d}$ & $27,87 \mathrm{~d}$ & $14,4 \mathrm{~d}$ \\
\hline
\end{tabular}

Dans une même colonne, les moyennes suivies de la même lettre ne diffèrent pas significativement selon le test de Newman-Keuls au seuil de $5 \%$.

F0 = témoin (terre tout venant) ; F2 = pelure de banane séchée et broyée ; F3 = 75\% F2 + $25 \%$ compost de fiente de poules ; F4 = $50 \%$ F2 + $50 \%$ compost de fiente de poules ; F5 = $25 \%$ F2 + $75 \%$ compost de fiente de poules. Effets des mélanges sur les paramètres de
croissance du bananier plantain

Hauteur du pseudo-tronc: Le taux de croissance absolue en hauteur du pseudo-tronc a varié de 8,13 à $18,51 \mathrm{~cm}$ en fonction du traitement appliqué (Figure 3).
Les valeurs les plus élevées ont été obtenues avec les traitements F2 et F3. Avec une valeur de $8,13 \mathrm{~cm}$, le traitement F1 est celui qui a induit le plus faible taux de croissance.

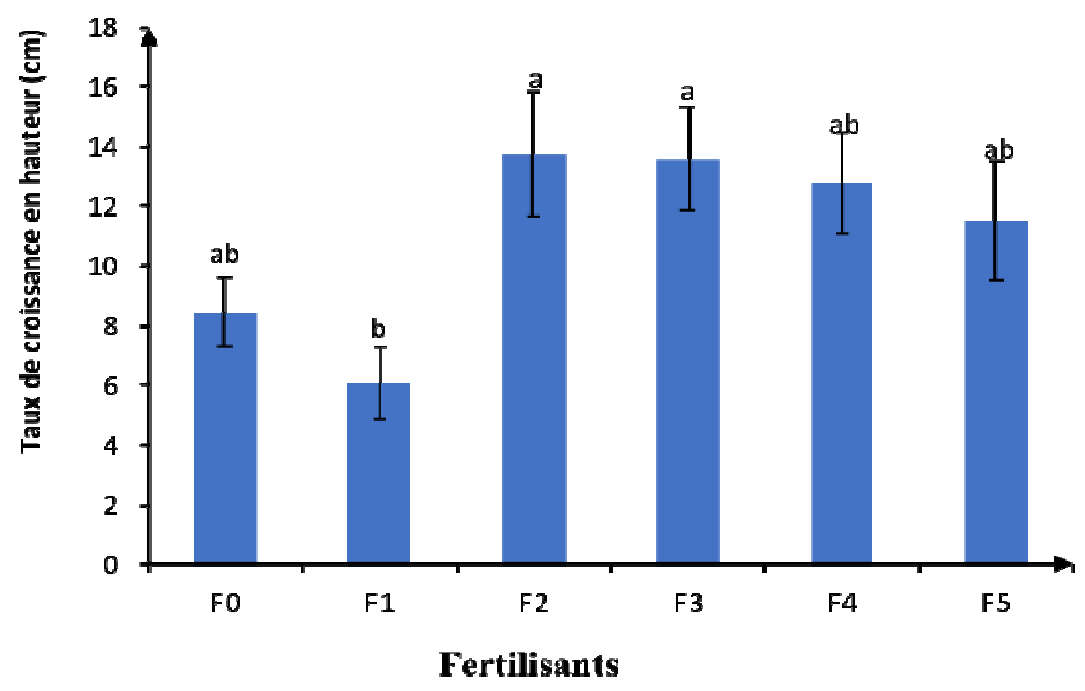

Les moyennes affectées de lettres différentes sur les histogrammes sont significativement différentes au seuil de $5 \%$ (Test de Newman-Keuls).

Figure 3 : Taux de croissance absolue en hauteur du pseudotronc de bananier plantain Big Ebanga en fonction des fertilisants appliqués.

Circonférence du pseudo tronc et Robustesse des plants de bananier plantain $(H / C)$ : Les valeurs moyennes relatives à la circonférence du pseudo-tronc ont varié de - 0,17 à $2,93 \mathrm{~cm}$ en fonction des traitements appliqués (Figure 4). Une différence significative a été observée entre les moyennes $(p<5 \%)$. Les taux de croissance radiale absolue les plus élevés ont été observés avec les fertilisants F2, F3, F4 et F5, dont les valeurs respectives ont été 2,$93 ; 2,91 ; 2,73$ et $2,61 \mathrm{~cm}$ contre un taux négatif observé avec le fertilisant $F 1$. Le ratio de robustesse des jeunes plantes de bananier plantain qui a varié de 3,57 à 3,87 en fonction des traitements appliqués n'a pas été significativement marqué (Figure 5). 


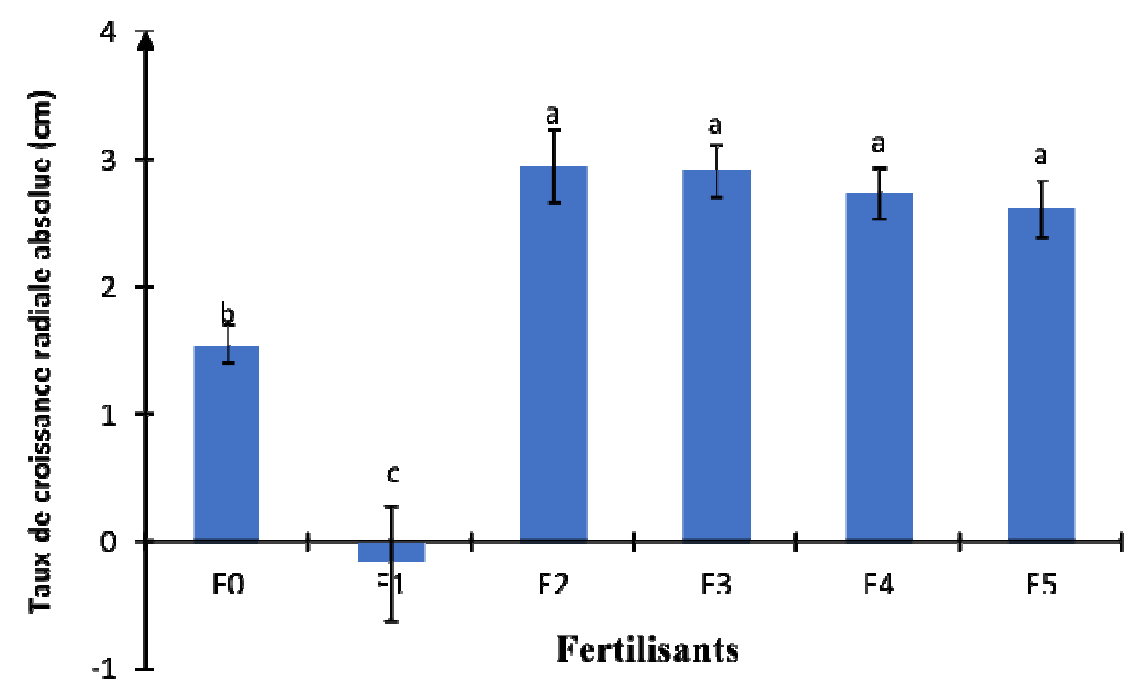

Les moyennes affectées de lettres différentes sur les histogrammes sont significativement différentes au seuil de $5 \%$ (Test de Newman-Keuls).

Figure 4 : Taux de croissance radiale absolue des plants de bananier plantain Big Ebanga en fonction des fertilisants appliqués.

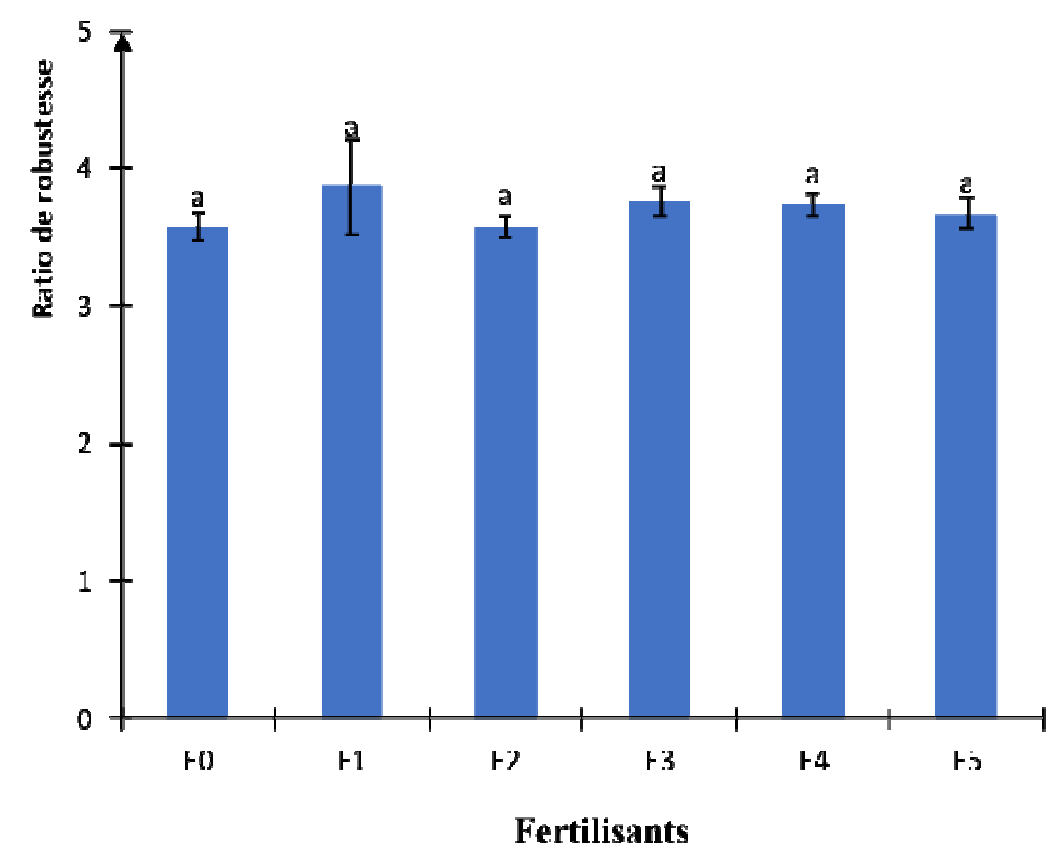

Les moyennes affectées de lettres différentes sur les histogrammes sont significativement différentes au seuil de $5 \%$ (Test de Newman-Keuls).

Figure 5 : Ratio de robustesse des plants de bananier plantain Big Ebanga en fonction des fertilisants appliqués

Nombre de feuilles : Le nombre de feuilles émises par les plants de bananier a varié de façon significative $(p<5$ $\%$ ) suivant le traitement fertilisant entre 5,12 et 7,63 (Figure 6). Les fertilisants F2, F3, F4 et F5 ont induit plus de feuilles par rapport au témoin F0 et au NPK (F1). L'analyse en composante principale (Figure 7) réalisée a permis de traiter simultanément les paramètres de croissance et la dispersion des fertilisants sur la base de la réponse des plants de bananier plantain Big Ebanga après application. Les axes 1 et 2 contribuent pour 95,85 $\%$ à la variation totale observée et expliquent au mieux la dispersion et le classement de ces fertilisants. Les 
paramètres de croissance sont fortement et positivement corrélés à l'axe 1. La dispersion des fertilisants dans le plan 1 et 2 a permis d'obtenir trois (3) groupes de fertilisants dont: ceux qui ont induit une meilleure croissance des plants de bananier plantain (F2, F3, F4 et $\mathrm{F5})$, $\mathrm{F} 0$ qui a induit une croissance moyenne et $\mathrm{F} 1$ qui a induit une faible croissance des jeunes plantes de bananiers plantain Big Ebanga.

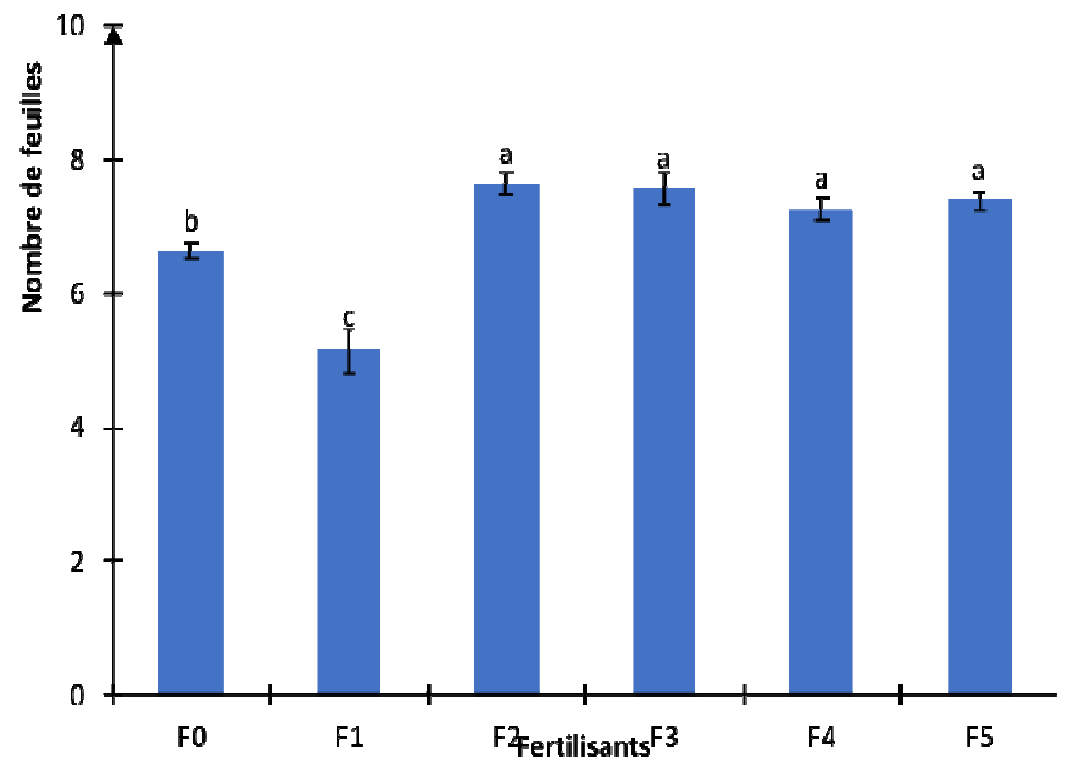

Les moyennes affectées de lettres différentes sur les histogrammes sont significativement différentes au seuil de $5 \%$ (Test de Newman-Keuls).

Figure 6: Nombre de feuilles de bananier plantain Big Ebanga en fonction des fertilisants appliqués

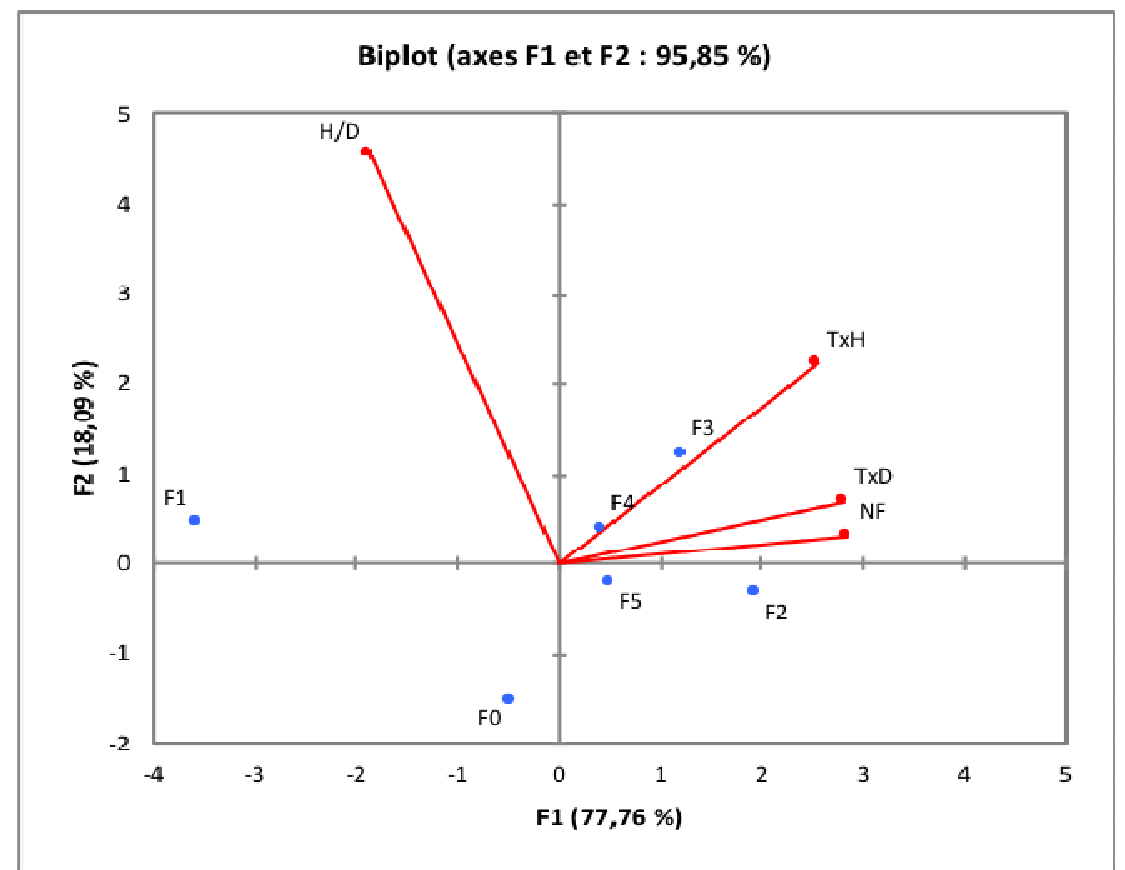

Figure 7 : Répartition des fertilisants et des paramètres d'évaluations de la croissance des plants de bananier plantain Big Ebanga dans le plan 1 et 2 d'une analyse en composante principale 


\section{DISCUSSION}

L'analyse des résultats relatifs à la composition minérale des formulations a montré d'une part que les fertilisants F4 et F5 sont plus riches en azote (N), phosphore (P), calcium $(\mathrm{Ca})$ et sodium $(\mathrm{Na})$ et d'autre part que les fertilisants F2 et F3 sont plus riches en potassium (K). L'analyse en composante principale (ACP) a permis de les caractériser en trois groupes, sur la base de leur teneur en minéraux. La forte variation de la composition minérale des différentes formulations testées est due à leur hétérogénéité. Les fertilisants F2 et F3 sont plus riches en potassium. Leur richesse en ce minéral pourrait s'expliquer par leur forte proportion en pelure de banane. Selon Biego et al. (2010), le potassium provient essentiellement des nervures des peaux mûres du plantain. Les fertilisants F4 et F5 sont plus riches en minéraux par rapport aux autres fertilisants. Cette différence est due à un effet additionnel de la fiente de poules à la pelure de banane plantain. Ces résultats confirment ceux de Demir et al. (2010) et Delago et al. (2012) qui ont montré que le fumier de volailles est un produit très concentré en éléments fertilisants (azote, potasse et phosphore et en oligo-éléments). Concernant les caractéristiques chimiques des mélanges, la valeur du $\mathrm{pH}$ des différentes formulations est très variable. Cette variabilité proviendrait de la composition des intrants et de l'intensité de nitrification de ces produits organiques pendant le stockage (Larney et Angers, 2012). Les fertilisants $\mathrm{F} 4$ et $\mathrm{F} 5$ présentent des valeurs de $\mathrm{pH}$ qui sont proches de la neutralité $(\mathrm{pH} 7)$. A ce $\mathrm{pH}$, les éléments minéraux contenus dans le sol sont mis à disposition de la plante $(F A O, 2005)$. Suite à la caractérisation chimique des différentes formulations, les résultats ont montré que le taux de matière organique (MO) est relativement faible pour le fertilisant $\mathrm{F} 5$ et le témoin $\mathrm{F} 0$, mais supérieur à 50 $\%$ pour les fertilisants $\mathrm{F} 2, \mathrm{~F} 3$ et $\mathrm{F} 4$. Cette variation de la teneur en matière organique pourrait être attribuée à la nature des intrants et au degré de minéralisation de ces fertilisants au moment de l'analyse. En mélange avec la PBSB, les proportions croissantes de compost de fiente de poules ont tendance à diminuer le taux de MO. En effet, le fumier de volailles, grâce à sa forte minéralisation, affiche une teneur en $\mathrm{MO}$ basse, due à sa pauvreté en composés ligneux (Azeez et Van Averbeke, 2010). Les résultats relatifs au rapport $\mathrm{C} / \mathrm{N}$ ont montré qu'il est très élevé $(C / N>20)$ pour les fertilisants $F 2$ et $F 3$ et moins élevé $(C / N<20)$ pour les fertilisants $F 4$ et $F 5$. Cette différence pourrait s'expliquer par la variabilité des intrants, par leur degré de minéralisation et surtout par leurs teneurs en azote. Le rapport $\mathrm{C} / \mathrm{N}$ est fréquemment utilisé pour évaluer le processus de minéralisation de la matière organique (Bernal et al., 1998). Les fertilisants F2 et F3 ont une minéralisation très lente, donc libèrent lentement les éléments nutritifs qu'ils contiennent. Sur l'aspect qualité agronomique du fertilisant organique, Alexander (1994) souligne qu'un rapport C/N entre 15-20 est considéré comme optimum. De ce fait, les fertilisants $\mathrm{F} 4$ et $\mathrm{F} 5$ présentent une meilleure minéralisation, donc ils libèrent beaucoup plus vite l'azote pour le mettre à la disposition des plantes. Par rapport à l'effet des mélanges sur la croissance des rejets écailles, l'évaluation de la hauteur et de la circonférence des pseudos tiges permet d'apprécier la croissance des plants (Randrianarison, 2004 ; Tshimbombo et al., 2018), favorisée par l'azote qui joue un rôle de premier plan. L'ACP réalisée a permis certes de caractériser les fertilisants organiques testés en deux classes sur la base de leurs teneurs en minéraux, mais ils ont eu le même effet sur la croissance des bananiers plantain. En effets, les fertilisants F2, F3, F4 et F5 ont induit une meilleure croissance des bananiers plantain par rapport au témoin F0 et le NPK. Cette croissance des bananiers pourrait s'expliquer par la bonne teneur en minéraux et les bonnes propriétés chimiques de ces fertilisants. Les éléments nutritifs en qualité et en quantité ont donc été mis à la disposition des bananiers, notamment l'azote, le phosphore et le potassium. L'absorption des éléments minéraux par les bananiers serait facilitée par le $\mathrm{pH}$ basique de ces fertilisants organiques. Selon Mérelle (1998), un pH basique favorise une meilleure absorption racinaire des éléments nutritifs nécessaire à la croissance de la plante. Le nombre de feuilles chez les plants traités avec les fertilisants F2, F3, F4 et F5 est plus élevé par rapport au traitement témoin et le NPK. Ces résultats pourraient s'expliquer par la teneur en azote, principal facteur de croissance des végétaux verts. En effet, l'azote est un facteur essentiel de la croissance des plants, surtout au niveau des tiges et des feuilles (Anonyme 1, 2002; Nyembo et al., 2012). Contrairement aux fertilisants organiques, le témoin F0 et le NPK ont induit une faible croissance des bananiers plantains. L'application du NPK a provoqué la brûlure des plants de bananiers. Cette observation est en concordance avec celle de Omari et al. (2012). Ces auteurs ont observé une réduction des paramètres de croissance des portes greffes d'agrumes après un apport d'azote à la dose de $10 \mathrm{mM}$. Cette réduction de la croissance des plantules serait occasionnée par une augmentation de la pression osmotique du sol engendré par un apport d'azote en grande quantité. Le jaunissement de feuille, le dépérissement de la tige et la mort des plants ont été 
observés dans le lot de plants traités avec le NPK. Ces symptômes pourraient être associés à l'apport d'une dose trop importante. Cet excès aurait donc entrainé une acidification de l'environnement racinaire. En effet, la

\section{CONCLUSION ET PERSPECTIVES}

La présente étude a permis de mettre en évidence l'effet des résidus organiques sur la croissance en pépinière de rejets écailles de bananiers plantain de la variété Big Ebanga. L'analyse minérale de ces mélanges fertilisants a montré leur richesse en $\mathrm{N}, \mathrm{P}, \mathrm{K}, \mathrm{Mg}$, Na et $\mathrm{Ca}$. Les données de croissance ont varié en fonction des fertilisants appliqués. Les meilleures formulations sur les paramètres de croissances ont été F2, F3, F4 et F5. L'analyse en composantes principales a montré que la pelure de banane plantain séchée et broyée (PBSB) utilisée simple ou en mélange avec le compost de fiente de poules (CFP) induit une croissance harmonieuse des

\section{REFERENCES BIBLIOGRAPHIQUES}

Alexander M, 1994. Introduction to Soil Microbiology. John Wiley Eds. Library of congress catalog card number: 61-11512.

Anonyme 1, 2002. Ministère des Relations Extérieurs, de la Coopération et du Développement, Momento de l'Agronome (7 edn). MRECD: Paris, $379 p$.

Aowad M. et Mohamed A, 2009. The effect of bio, organic and mineral fertilization on productivity of sunflower seed and oil yields, Kafr El Sheikh University, J. Agric. Res., 35 (4) : 1013-1027.

Azeez J.O. et Van Averbeke W, 2010. Nitrogen mineralization potential of three animal manures applied on a sandy clay loam soil. Bioresource technology, 101 (14): 5645-5651.

Bernal MP, Sanchez-Monedero MA, Paredes $C$ et Roig A, 1998. Carbon mineralization from organic wastes at different composting stages during their incubation with soil. Agriculture, Ecosystems \& Environment, 69 (3) : 175-189.

Biego G, Koffi K, Chatigre K, N'doume C et Kouadio L, 2010. Détermination des minéraux de sousproduits de cultures d'exportation et vivrières de Côte d'Ivoire. Journal sciences pharmaceutiques et biologiques, 12 (2) : 13-24.

CNRA (Centre National de recherche Agronomique), 2016. Bien cultiver la banane plantain en Côte d'Ivoire, Centre National de Recherche Agronomique. Fiche technique du bananier plantain, $4 \mathrm{p}$.

Delago M, Rodriguez C, Martin JV, De Imperial RM, et Alonso F, 2012. Environmental assay on the conversion d'une molécule d'urée en nitrate entraine une libération de deux protons. L'accumulation de ces ions a pour conséquence une acidification de la rhizosphère (Le Gall, 2007).

jeunes plantes de bananier plantain. II ressort de cette étude que la pelure de banane séchée et broyée peut être recommandée aux agriculteurs parce que riche en minéraux, accessible, moins coûteux et simple à réaliser. Sa vulgarisation pourrait contribuer d'une part à la pratique de l'agriculture biologique et d'autre part à l'assainissement de l'environnement urbain. En perspectives, il serait judicieux de conduire l'étude jusqu'à la production des fruits; d'effectuer une application de ces produits sur une grande échelle de culture de bananes ; enfin, d'évaluer l'impact de ces produits sur les propriétés physico-chimiques des sols.

effect of poultry manure application on soil organisms in agroecosystems. Science of the total environment, 416: 532-535.

Demir K, Sahin O, Kadioglu YK, Pilbeam DJ et Gunes A, 2010. Essential and non essential element composition of tomato plants fertilized with poultry manure. Scientia horticulturae, 127 (1) : 16-22.

Diallo L., 2002. Effet de l'urée et du fumier sur le rendement du maïs. Mémoire d'ingénieur du Développement Rural / Option Agronomie. IDR / UPB. Burkina Faso. 54 p.

FAO (Organisation des Nations Unies pour l'alimentation et l'agriculture, Food and Agriculture Organization), 2010. Les répercussions du changement climatique sur la sécurité alimentaire et la gestion des ressources naturelles en Afrique. Document de référence rédigé pour la vingt-sixième Conférence régionale pour I'Afrique. Luanda, Angola, 3-7 mai. ARC/10/8.

FAO, 2005. Notion de nutrition des plantes et de fertilisation des sols. Manuel de formation, Projet Intrants, Niger, $24 \mathrm{p}$.

FAOstat, 2017. Division de la statistique de l'Organisation des Nation Unies pour l'Alimentation et l'Agriculture.

http://www.fao.org/faostat/fr/\#data/QC.

Foure E. et Tezenas DM, 2000. Les productions bananières : un enjeu économique majeur pour la sécurité alimentaire. Bulletin de liaison de la 
coopération régionale pour le développement des produits horticoles en Afrique, $18: 23-28$.

Goyal S, Dhull, SK et Kapoor KK, 2005. Chemical and biological changes during composting of different organic wastes and assessment of compost maturity. Bioresource technology, 96(14) : 1584-1591.

Igoud S, Tou I, Kehal S, Mansouri N et Touzi A, 2002. Première approche de la caractérisation du biogaz produit à partir des déjections bovines. Rev. Energ. Ren., 4 : 123-128.

Jones D., 2000. Introduction to banana, Abaca and Ensete. CABI, London, Wollong Ford, UK: 1-31.

Larney F.J. et Angers DA, 2012. The role of organic amendments in soil reclamation: A review. Canadian Journal of Soil Science, 92 (1): 19-38.

Lassois L, Busogoro J-P et Jijakli H, 2009. La banane : de son origine à sa commercialisation. Biotechnol. Agron. Soc. Environ., 13 (4) : 575586.

Le Gall A.C., 2004. Effets des dépôts atmosphériques de soufre et d'azote sur les sols et les eaux douces en France. INERIS, $112 \mathrm{p}$.

Mérelle F., 1998. L'analyse de terre aujourd'hui. Nantes, France : Édition GEMAS, Nantes. $184 \mathrm{p}$.

M'Sadak Y., Ben M'Barek A, 2015. Characterization of Co-products of the pilot digesters to animal biomass in Tunisia. J. Fundment. Appl. Sci. 7 : 185-202.

Moughli L., 2000. Les engrais minéraux; Caractéristiques et Utilisations. Bulletin mensuel d'information et de liaison du Programme National de Transfert de Technologie en Agriculture (PNTTA), Institut Agronomique et Vétérinaire Hassan II, Septembre 72, $4 \mathrm{p}$.

Mulaji K., 2011. Utilisation des composts de bio-déchets ménagers pour l'amélioration de la fertilité des sols acides de la province de Kinshasa (République Démocratique du Congo). Thèse Université de Liège, Belgique, 191 p.

Nyembo KL, Useni SY, Mpundu MM, Bugeme MD, Kasongo LE et Baboy LL, 2012. Effets des apports des doses variées de fertilisants inorganiques (NPKS et Urée) sur le rendement et la rentabilité économique de nouvelles variétés de Zea mays L. à Lubumbashi, Sud-Est de la RD Congo. Journal of Applied Biosciences, 59: 4286-4296.

Omari FE, Beniken L, Gaboune F, Zouarhi A, Benkirane $\mathrm{R}$ et Benyahia $\mathrm{H}, 2012$. Effet de la nutrition azotée sur les paramètres morphologiques et physiologiques de quelques porte-greffes d'agrumes. Journal of Applied Biosciences, 53: 3773-3786.

Rabbani G, Teka T, Zaman B, Majid N, Khatum M et Fuchs $G, 2001$. Chemical studies in persistent diarrhea: dietary management with green banana or pectin in Bangladeshi children. Gastroenterology, 121 (3) : 554-560.

Randrianarison N., 2004. Essais de conduite de pépinière en tabaculture de décrue semis sur plates-bandes et sur mini-pots. Détermination des effets de différents types de substrat sur le taux de germination. Détermination de la méthode de faucillage la plus appropriée. Mémoire de fin d'études en vue de l'obtention du diplôme d'ingénieur agronome. Option : Agriculture. École supérieure des sciences agronomiques, département agriculture. Université d'Antananarivo, $118 \mathrm{p}$.

Tshimbombo J, Kankolongo M, Tshizembe M, Bongali B, Banga Banga $M$, Kasongo $K$, Badibanga $M$ et Mbaji KN, 2018. L'influence des fertilisants organiques liquides D.I. GROW et inorganiques NPK 17-17-17 + Urée sur le rendement et la rentabilité de la culture du maïs à Ngandajika. Journal of Applied Biosciences, 122 : 1225612261.

Turquin L, Aké S et Anno P, 2007. Effets des dérives auxiniques sur la production du bananier plantain cv Corne 1 en Côte d'lvoire. Sciences et Techniques, sciences naturelles et agronomie, $29(1-2): 105 \mathrm{p}$.

Useni S, Baboy L, Nyembo K et Mpundu M, 2012. Effets des apports combinés de biodéchets et de fertilisants inorganiques sur le rendement de trois variétés de Zea mays $L$. cultivées dans la région de Lubumbashi. J. Appl. Biosci. 54 : 3935-3943.

Yao N, Atsin GJO, Gnonhouri P, Traore S, Kobenan K et Tahouo O, 2014. Nouveaux systèmes de production de plantain en Côte d'Ivoire. In : le point sur le CNRA en 2014, 10-12.

Yerima BPK., Tiamgne AY et Van Ranst E, 2014. Réponse de deux variétés de tournesol (Helianthus sp.) à la fertilisation à base de fiente de poule sur un Hapli-Humic Ferralsol du Yongka Western Highlands Research garden park (YWHRGP) Nkwen-Baamenda, Cameroun, Afrique central. Tropicultura. 32(4):168-176. 